\title{
Near patient testing: the challenge for clinical pathology
}

\author{
D J Goldie, H Kemp
}

\section{Introduction}

Near patient testing (NPT) is not new: it developed from the technological advances in the late $70 \mathrm{~s}$ and $80 \mathrm{~s}$, was the subject of a major conference, ${ }^{1}$ and has been much discussed and written about since..$^{2-6}$ It has not, until recently, however, made a major impact on the workload or activities of most pathology laboratories in the United Kingdom. This less rapid than predicted development can perhaps largely be attributed to financial constraints but is no doubt part attributable to a lack of enthusiasm among many laboratory workers who viewed such developments as a threat to their professional activities.

We believe that clinical pathologists must now embrace NPT because it will become more widespread, and clinical pathologists have a professional responsibility to see that it is done in the best possible manner to deliver the maximum advantage to patient care.

\section{Background}

Individuals and organisations have promoted NPT for a variety of reasons, some more worthy than others. In our view the prime justification for moving an analytical procedure from the laboratory to the bedside or clinic is timeliness. An example of this is when information is needed to make rapid adjustments to patient treatment, such as decisions made on the basis of blood gas analysis results in an intensive care or special care baby unit. There will also be occasions when the laboratory is unable to provide a sufficiently timely service to outpatient clinics or general practitioners' surgeries. Such a failure may necessitate a further appointment, wasting scarce health care resources and patients' time. Furthermore, there is an increasing appreciation of the value of the rapid provision of results to allay patient anxieties in our consumer driven culture. There is also a strong case to be made for encouraging diabetic patients to undertake home monitoring, not only for their convenience and the immediate availability of the results, but to avoid the recently described phenomenon of "white coat hyperglycaemia" in which clinic glucose concentrations are not representative of diabetic control because of an increase in blood glucose associated with the stress of a hospital visit.

Other pressures for the development of NPT result from commercial considerations: a proliferation of analytical equipment is profitable to equipment manufacturers and sup- pliers of reagents, disposables, and quality control materials. Furthermore, if the provider of an NPT service is remunerated on a fee per item of service basis this may be an incentive to promote testing which is not necessarily in the patient's best interest. We view the development of the "High Street" measurement of serum cholesterol concentration as a good example of this phenomenon, a view shared by others. ${ }^{8}$ Although it can be argued that some interests are attempting to promote NPT and home testing for financial gain, and while there is no doubt that the direct costs are higher, it must be conceded that the balance between these and the socalled macro economics is not clear. ${ }^{9}$

Clinical pathologists must recognise that NPT may be promoted for both laudable and professionally supportable reasons as well as for other reasons. The profession must be careful to try to make these distinctions so that they may discourage, as far as they are able, inappropriate NPT developments.

Overall, therefore, the clinical pathologist should approach NPT with care but, when it is recognised to be in the best interests of providing a better service to the patient, the laboratory worker has a clear professional responsibility to ensure that the service provided is of as high a standard as possible.

\section{Difficulties of NPT}

The difficulties associated with the provision of an acceptable quality NPT testing service are very much the same as those associated with the provision of a good laboratory service, and it is important that this is always borne in mind. Dual standards are simply not acceptable and NTP must be undertaken with the same attention to the needs of the patient and the provision of a safe working environment for the service provider as would be required in a laboratory setting.

The main considerations that need to be borne in mind in the establishment of acceptable NPT arrangements are as follows:

(1) Selection of instrumentation appropriate for the purpose and the provision of satisfactory arrangements for its maintenance, and back-up arrangements in the event of machine failure.

(2) Arrangements for performing the analyses must be such that a machine is operated only by those who have been trained to an acceptable standard and who observe agreed procedures of analysis including procedures for quality control and result 
recording. Medicolegal considerations of responsibility for an erroneous result and the Data Protection Act must be borne in mind when establishing these operating procedures.

(3) All NPT must be conducted under the same criteria for safety as would apply for the protection of both staff and patients in a conventional laboratory. The requirements of acts such as the Health and Safety at Work Act, 1974, the Consumer Protection Act 1987, the COSSH regulations, 1988, and the requirements of the Safe Working and the Prevention of Infection in Clinical Laboratories Code 1991 are not suspended however small a "laboratory" may be.

(4) The provision of a good laboratory service entails more than the mere provision of an analytical service, in particular the availability of professional guidance in the selection and interpretation of tests must also be an integral part of the arrangements.

It is apparent that attention to all the above requirements represents a considerable professional challenge, and there is no doubt that clinical pathologists are uniquely placed to meet it. This has already been recognised in the case of extra laboratory blood glucose monitoring where the then DHSS issued a Hazard Notice ${ }^{10}$ following a failure of proper patient care which included specific advice on the involvement of laboratories in NPT.

\section{The future}

The fact that NPT is now with us, will grow, and needs to be demonstrably done properly and safely is being increasingly recognised. Very clear guidelines have been issued by the Joint Working Group on Quality Assurance. ${ }^{11}$ In these guidelines the crucial role of the laboratory is very clearly defined in the statement that "Laboratory staff should take responsibility for the near patient service in a manner agreed between the Heads of the appropriate laboratory and clinical services or directorates". The document gives clear advice on the role of the laboratory in all aspects of NPT and urges that similar arrangements must also be made where diag- nostic instruments are used in general practices and elsewhere-for example, industrial medical centres, pharmacies, etc. It remains to be seen how far this policy of laboratory involvement can, in practice, be carried but the philosophy is not in any doubt.

At present there is little a concerned clinical pathologist can do if colleagues choose not to cooperate, but this situation is likely to change rapidly in the near future. In the first place the process of accreditation of laboratories in the United Kingdom has started under the aegis of CPA (UK) Ltd. In the most recent newsletter of that organisation it is clearly stated that CPA (UK) Ltd endorses the guidelines of the Joint Working Group on Quality Assurance and any department wishing to gain CPA accreditation must comply with them. ${ }^{12}$ Secondly, it is to be expected that as accreditation becomes more widely sought and accepted, purchasers of laboratory services will increasingly insist that contracts be placed only with accredited laboratories. Thus the circle will be complete and clinical pathologists will need to meet the challenge.

1 Marks V, Alberti KGMM, eds. Clinical biochemistry nearer the patient Edinburgh: Churchill Livingstone, 1985.

2 Marks V. Essential considerations in the provision of nearpatient testing facilities. Ann Clin Biochem 1988;25 220-5.

3 Farr AD. Near-patient 'laboratory' testing. Med Lab Sci 1990;47:249-50.

4 Chernow B. The bedside laboratory. Chest 1990;97 (Supplement): 183S-213S.

5 Burritt MF. Current analytical approaches to measuring blood analytes. Clin Chem 1990;36(B):1562-6.

6 Meyerhoff ME. New in vitro analytical approaches for clinical chemistry measurements in critical care. Clin Chem 1990;36(B):1567-72.

7 Campbell LV, Ashwell SM, Borkman M, Chisholm DJ White coat hyperglycaemia: disparity between diabetes clinic and home blood glucose concentrations. $B M \mathcal{F}$ 1992;305:1194-6.

8 Anonymous. Home cholesterol testing [Editorial]. Lancet 1992;340:1386.

9 Craig TM. The economics of near patient testing. In Marks V, Alberti KGMM, eds. Clinical biochemistry nearer the patient. Edinburgh: Churchill Livingstone, nearer the patie

10 DHSS. Blood glucose measurements: reliability of results produced in extra-laboratory areas. London: DHSS, 1987. HN (Hazard) (87) 13.

11 Joint Working Group on Quality Assurance. Guidelines on the control of near-patient tests (NPT) and procedures performed on patients by non-pathology staff. 1992. Available from D Kilshaw, Secretary, JWGQA. c/o Mast House, Derby Road, Liverpool, L20 1EA.

12 Clinical Pathology Accreditation (UK) Ltd. Bulletin No 3. Sheffield, CPA: 1992. 